\title{
Sequência de Möbius: protocolo de anamnese e avaliação - relato de caso
}

\section{Möbius Sequence: anamnesis and evaluation protocol - case report}

\author{
Tatiana Cantarelli Andrade Lima de Albuquerque ${ }^{1}$, Renata Ramalho da Silva Barreto ${ }^{2}$, \\ Thieza Christy Carregã Martins da Costa ${ }^{3}$, Zelita Caldeira Ferreira Guedes ${ }^{4}$
}

\begin{abstract}
RESUMO
A Seqüência de Möbius (SM) foi inicialmente descrita por Von Graefe em 1888. Caracteriza-se por apresentar paralisia do VI e VII pares cranianos, determinando a ausência de expressão facial acompanhada de estrabismo ocular convergente. Nas crianças avaliadas deste grupo encontramos todas as características descritas. Entretanto, podem ocorrer paralisias ocasionadas por outros pares cranianos dificultando ou impossibilitando a mobilidade de língua e palato mole, com dificuldades na produção dos fonemas e/ou excesso de nasalização na emissão dos mesmos. O presente estudo teve como objetivo divulgar o protocolo utilizado em nossa Instituição com pacientes com SM e relatar um caso atendido neste serviço, como mera exemplificação. O protocolo consta de duas partes: anamnese e avaliação das estruturas e das funções estomatognáticas. A anamnese consiste na aplicação de questionário aos responsáveis, para investigar toda a história do paciente, desde a gestação até o presente momento. Na avaliação fonoaudiológica específica para o sistema estomatognático são investigados mobilidade, motricidade, tônus e postura dos órgãos fonoarticulatórios e atividades das funções neurovegetativas. Há também a investigação dos músculos responsáveis pela expressão facial que são avaliados individualmente, para se ter a real possibilidade de manifestação desses pacientes. Para exemplificação do protocolo, com ambas as partes, um paciente do sexo masculino com 10 anos de idade foi avaliado e os resultados estão aqui apresentados. O paciente faz terapia nesta Instituição desde o ano de 2000. Neste protocolo procuramos identificar tanto as manifestações fonoaudiológicas características da $\mathrm{SM}$, bem como descrever as particularidades individuais.
\end{abstract}

Descritores: Protocolo; Avaliação; Anamnese; Síndrome de Möbius/diagnóstico; Sistema estomatognático; Relatos de casos

\section{INTRODUÇAO}

A Sequiência de Möbius (SM) é caracterizada basicamente pelo comprometimento dos nervos abducente e facial (VI e VII pares, respectivamente) e conseqüente paralisia, quase sempre bilateral, dos músculos inervados por estes pares, resultando numa alteração importante de mímica facial e na impossibilidade de movimentar o globo ocular lateralmente ${ }^{(1)}$.

Outros pares cranianos também podem estar comprometidos (III, IV, V, IX, X e XII) e anomalias tais como micrognatia, defeitos de membros, tórax, deficiência mental, malformações cardíacas e do trato urinário também podem ser observados.

Trabalho realizado no Ambulatório de Paralisia Facial Congênita da Disciplina de Distúrbios da Comunicação Humana da Universidade Federal de São Paulo - UNIFESP - São Paulo (SP), Brasil.

(1) Fonoaudióloga especializada do Departamento de Fonoaudiologia da Universidade Federal de São Paulo - UNIFESP - São Paulo (SP), Brasil.

(2) Fonoaudióloga especializada do Departamento de Fonoaudiologia da Universidade Federal de São Paulo - UNIFESP - São Paulo (SP), Brasil.

(3) Fonoaudióloga voluntária do Departamento de Fonoaudiologia da Universidade Federal de São Paulo - UNIFESP - São Paulo (SP), Brasil.

(4) Doutora, Professora Associada do Departamento de Fonoaudiologia da Universidade Federal de São Paulo - UNIFESP - São Paulo (SP), Brasil. Endereço para correspondência: Renata Ramalho da Silva Barreto. R. Giuseppe Uliane, $\mathrm{s} / \mathrm{n}^{\circ}$, casa 50, Demarchi, São Bernardo do Campo - SP, CEP 09820-160. E-mail: renata_ramalho@ hotmail.com

Recebido em: 17/12/2007; Aceito em: 14/12/2008
Tal doença caracteriza-se também por alterações de extremidades, como pés tortos congênitos, sindactilias (dedos unidos) e adactilias (ausência de dedos das mãos e/ou pés) ${ }^{(2-6)}$.

A ressonância hipernasal durante a fala é muito comum em pacientes com SM. A paralisia ocasionada por outros pares cranianos, muitas vezes dificulta ou até mesmo impossibilita a mobilidade da língua e do palato mole ocasionando dificuldades na produção dos fonemas e/ou o excesso de nasalização na emissão dos mesmos.

A paralisia facial compromete as atividades voluntárias, automáticas e reflexas dos lábios que estão diretamente relacionados com a fala ${ }^{(7)}$. Frente a este dado, pode-se deduzir que a fala do indivíduo que nasce com a SM será afetada em maior ou menor grau.

As crianças com SM possuem prejuízos articulatórios de fala e alterações importantes da voz. A qualidade vocal muitas vezes apresenta alterações significativas como, rouquidão crônica, nódulos nas pregas vocais, voz aspirada e com baixa intensidade (em decorrência da hipernasalidade) e a voz soprosa, que são a tentativa frustrada de corrigir o excesso de nasalidade.

Devido ao grande número de anomalias que os indivíduos portadores da SM podem apresentar, seu tratamento deve ser multidisciplinar, sendo que o papel do fonoaudiólogo é tentar minimizar as dificuldades desses indivíduos. 
Temos padronizado a anamnese e avaliação do sistema estomatognático realizada com esses pacientes, com a finalidade de conhecer de forma mais profunda, os diferentes detalhes que cada um apresenta na heterogeneidade de manifestação ${ }^{(8)}$.

Por se tratar de uma Síndrome rara, é de suma importância a padronização de protocolos de anamnese e avaliação bem elaborados, constando de informações e dados minuciosos para chegarmos a uma hipótese diagnóstica fonoaudiológica precisa.

\section{APRESENTAÇÃO DO CASO CLÍNICO}

Com o ensejo de apresentar os protocolos usados no atendimento desses pacientes em nossa Instituição, passaremos a apresentá-los e, visando melhor entendimento, usaremos as respostas obtidas na avaliação de um paciente como exemplo.

Participou do presente estudo um paciente do sexo masculino, de 10 anos de idade, com SM diagnosticada por neurologista ou geneticista.

O paciente pertence ao Ambulatório de Paralisia Facial Congênita da instituição onde é realizada terapia fonoaudiológica desde o ano de 2000. O paciente recebeu o Termo de Consentimento Livre e Esclarecido, sendo que este foi assinado pelo seu responsável. O trabalho recebeu autorização do Comitê de Ética para sua realização $\left(n^{\circ} 1221 / 03\right)$.

A primeira parte do protocolo (Anexos 1 e 2) consiste na aplicação da anamnese com os responsáveis, em que se terá a oportunidade de investigação de toda a história do paciente, desde a gestação, intercorrências, medicamentos, condições de parto, dados do desenvolvimento neuropsicomotor, fala e linguagem, audição, alimentação, sono, hábitos, histórico escolar, doenças, internações e cirurgias, acompanhamento com outras especialidades e orientações de qualquer ordem que já tenham sido recebidas.

- Gestação: como foi, quantas semanas durou a gestação, se houve intercorrências, se a mãe fez uso de medicamentos durante o período de gestação e por quanto tempo.

Essas perguntas são necessárias, pois a literatura indica que a SM pode ser causada pelo uso de medicamento misoprostol - conhecido comercialmente como Citotec $^{\circledR}$.

- Dados do nascimento: neste tópico pergunta-se como foi o parto, a nota do APGAR, peso e tamanho de nascimento e se houve alguma intercorrência.

Estas crianças podem apresentar outras alterações no Sistema Nervoso Central (SNC) que podem propiciar nota do Apgar baixa, e exigir intervenções durante e após o parto, como ressuscitação, uso de oxigênio, internações e uso de sonda prolongados, dentre outras.

- Dados do desenvolvimento:

a) Desenvolvimento neuropsicomotor - pergunta-se sobre controle cervical, quando sentou com e sem apoio, quando engatinhou e quando andou. Um grande número dessas crianças pode apresentar atraso neuropsicomotor e também malformações genéticas (pés tortos congênitos) dificultando a execução de alguns movimentos.

b) Desenvolvimento de fala - investiga-se quando a criança balbuciou e falou as primeiras palavras. Sendo que a paralisia do Nervo Facial afeta a musculatura da língua e dos lábios.

c) Audição - pergunta-se se a criança ouve bem, assusta-se com barulho, acompanha os sons, teve otites ou se já realizou algum tipo de exame. Essas perguntas foram selecionadas de acordo com os relatos da literatura que indicam alterações auditivas. Este questionamento não exclui a realização de avaliação auditiva (protocolo que não será abordado neste artigo).

- Histórico alimentar: como a criança se alimenta e qual o tempo gasto para tal, se tomou ou toma mamadeira, qual o alimento ofertado na mamadeira, quem oferta a alimentação, qual a postura da criança durante a alimentação, a época das transições alimentares e como ocorreram. Como é oferecida alimentação, se por Via Oral (VO), Sonda Nasogástrica (SNG), Sonda Oro Gástrica (SOG) ou gastrostomia, qual a consistência e a quantidade do alimento e em que horários é oferecido, se há engasgos freqüentes e se perduram os hábitos orais.

Essas perguntas são feitas para saber como é suprida a ausência ou pouca movimentação da língua.

- Doenças: pergunta-se se a criança teve ou tem crises convulsivas, doenças respiratórias, Refluxo Gastroesofágico (RGE), alterações cardíacas, malformações e/ou alterações metabólicas, se faz uso de medicamentos e se fez alguma cirurgia.

Tal questionamento deve ser realizado, já que a SM às vezes apresenta associações com outras patologias, como Seqüência de Pierre Robin, calcificações cerebrais, e outras.

- Histórico escolar: que tipo de escola a criança freqüenta, como é o seu rendimento escolar e a sua interação com os colegas.

São feitas perguntas relacionadas à escolaridade devido à exclusão social sofrida pela falta de expressão facial.

- Acompanhamento com outras especialidades:

a) Oftalmologia - falta de movimentação do globo ocular e estrabismo congênito;

b) Cirurgia Plástica / Ortopedia - malformações congênitas, como os pés tortos e alterações de extremidades superiores;

c) Otorrinolaringologia / Fisioterapia - alterações respiratórias;

d) Cirurgião dentista - alterações dentárias;

e) Neurologia - verificar as possíveis alterações que acompanham a doença como convulsões, por exemplo;

f) Genética - investigação sobre se a origem da doença é genética, para que haja aconselhamento aos pais.

\section{Avaliação}

Depois de realizada a anamnese, a criança deve ser avaliada, quanto ao sistema estomatognático.

Investiga-se a mobilidade, motricidade, tônus e postura dos órgãos fonoarticulatórios (lábio, língua, bochechas, palato, mandíbula, maxila, dentes) e atividades das funções neurovegetativas (sucção, deglutição, mastigação e respiração). Além disso, os músculos responsáveis pela expressão facial 
são avaliados individualmente, para se ter dados sobre a real manifestação desses pacientes.

- Movimentação de cabeça: são solicitados movimentos de rotação e lateralização para verificar se os músculos envolvidos estão afetados.

- Face: observação do tipo facial.

- Movimentação dos olhos: avaliar que movimentos estão presentes durante a realização de olhar para cima e para baixo, de rotação e lateralização.

- Lábios e língua: posicionamento, tonicidade, mobilidade e motricidade, para avaliar até que ponto a musculatura encontra-se afetada pela paralisia.

- Articulação têmporo-mandibular (ATM) e mandíbula: movimentação (abertura de boca e lateralização da mandíbula), avaliação da condição de articulação de fala e alimentação.

- Bochechas: tonicidade, mobilidade e praxias orofacias.

- Mento: observação se há algum movimento compensatório na tentativa de se usar os lábios.

- Arcada dentária: estado de conservação, tipo de dentição, tipo de oclusão e linha média. São avaliadas as alterações dentárias de acordo com o descrito na literatura.

- Palato e véu do palato: são avaliadas estas estruturas para reconhecer o fechamento do Esfíncter Velofaríngico (EVF) nas funções de deglutição e fala.

- Funções neurovegetativas: as funções de mastigação, sucção, deglutição e respiração são avaliadas para verificar de que forma o paciente realiza estas funções, com ou sem compensações, já que muitos dos músculos envolvidos encontram-se paralisados.

\section{Relato dos resultados do paciente}

No protocolo mencionado, procuramos tanto identificar as manifestações fonoaudiológicas referentes ao sistema estomatognático características da SM como a descrever as particularidades individuais. Na avaliação dos 20 músculos/ grupos musculares responsáveis pelas expressões faciais, o paciente deve reproduzir o movimento após a demonstração do terapeuta. Assim, cada músculo é avaliado separadamente de acordo com sua função. Em relação ao sistema estomatognático, foram observadas as alterações estruturais e funcionais:

- Face: paralisia facial bilateral com predominância de movimento do lado esquerdo;

- Lábios: superior encurtado, inferior com eversão, ambos com alterações de tônus, mobilidade e postura - constantemente abertos; queda da comissura labial; acúmulo de saliva na região vestibular e de comissuras; frênulo labial encurtado;

- Articulação têmporo-mandibular: sem alterações;

- Mandíbula: com retrognatia, mobilidade alterada e com abertura de boca reduzida;

- Bochechas: hipertonia do lado direito, alteração de tônus e mobilidade (lado direito tônus aumentado, sulco naso labial acentuado, contração do mento na avaliação do lado esquerdo, contração do mento do lado esquerdo com movimentação de comissura para baixo).

- Mento: alteração de tônus - hipertônico;

- Dentes: débito na escovação, apinhamentos (canino + incisivo lateral direito);

- Arcada dentária: classe II, desvio de linha média para direita;

- Língua: hipertonia, presença de tremor, desvio para lado direito, alteração de mobilidade; freio normal;

- Palato: atresia;

- Véu do palato: movimentação normal, úvula e amídalas normais.

Quanto às funções neurovegetativas (funções primárias que garantem a sobrevivência - sucção, mastigação, deglutição, respiração) observou-se:

- Sucção: auxilio dos dentes incisivos centrais para manter o canudo e contração do mento. Pode-se classificá-la como predominantemente ineficiente caracterizada por: ausência de vedamento labial, preensão dento/línguo-dental do canudo, sem escape oral, com fraqueza, com ritmo e participação do bucinador.

- Mastigação: mordida inicial anterior, mordida lateral esquerda, movimentos rotatórios, mastigação bilateral com contração do masseter, sem vedamento labial (presença de contração do mento), pouca lateralização de língua, necessidade de empurrar o alimento com o dedo (após introdução do alimento na cavidade oral), formação do bolo coeso, centralização do bolo na língua e velocidade de mastigação diminuída.

- Deglutição: contato do incisivo central superior com lábio inferior eficiente, sem protrusão de língua, predominantemente sem movimentação de cabeça e sem interposição de lábios, sem vedamento labial, com contração de mento, com contração de masseter, com participação de musculatura perioral e propulsão adequada do bolo alimentar.

- Respiração: tipo costodiafragmática e modo misto (nasal e oral).

\section{DISCUSSÃO}

A SM foi descrita* como tendo a face com aspecto de máscara, com ausência de rugas de expressão, tanto no riso como no choro, além da impossibilidade para franzir a testa. Foi descrito também o escape de saliva no canto bucal constante, lábios permanentemente abertos, inabilidade para sorrir, microglossia, mobilidade de língua diminuída, palato duro em ogiva $^{(9)}$. Todas as alterações citadas pelo autor puderam ser encontradas em nosso paciente avaliado.

De acordo com alguns estudos ${ }^{(2-6)}$, dentre as alterações citadas, foi observado somente micrognatia, sendo descartadas todas as demais manifestações.

Quanto à mímica facial a avaliação demonstrou que a criança apresenta comprometimento dos músculos envolvidos na expressão facial. Pôde-se notar que há uma assimetria na execução dos movimentos ocasionada pela diferença do grau

* Möebius PJ. Ueber Infantilen Kernschwund, München Med. Wchnsehr. 35:91, 1888. 
de comprometimento dos pares cranianos, com mais movimento ao lado esquerdo. Na literatura, $\mathrm{SM}^{(1)}$ é caracterizada como uma paralisia facial bilateral, sem que haja comentário sobre a melhor eficiência de movimentos em um dos lados, como acontece nesse paciente.

Na SM observa-se freqüentemente dificuldade na deglutição, mastigação e no desenvolvimento da fala.

Durante a avaliação dos órgãos fonoarticulatórios, o paciente apresentou dificuldade na mastigação e deglutição desde a fase preparatória oral até a deglutição do bolo alimentar, devido as alterações anteriormente citadas.

No paciente, durante a avaliação do sistema sensório motor oral e da fala (levando-se em consideração apenas a mobilidade de execução dos sons) realizada, foi observada em sua articulação uma vogal sustentada fazendo com que em algumas vezes não conseguíssemos entender o que ele queria dizer. Fazia parte do protocolo, a repetição de sons isolados, palavras e frases, em que o paciente apresentou algumas dificuldades. Como foi relatado no parágrafo anterior, tal dificuldade é esperada levando em consideração a pouca mobilidade da língua e dos lábios na execução dos movimentos necessários para a produção dos sons da fala.

A impossibilidade ${ }^{(10)}$ de criar uma pressão intra-oral efetiva, ocasionando alterações articulatórias, pode ser observada em nosso paciente, além da distorção fonêmica.

Existem relatos $^{(11)}$ de que as alterações da mímica interferem no processo de comunicação favorecendo um mecanismo compensatório. O paciente avaliado também demonstrou essas alterações citadas pela autora, o que nos leva a pensar na dificuldade que ele tem em se relacionar com os outros, fazendo com que o mesmo se torne uma pessoa isolada.

Embora tenha sido apresentada a avaliação de apenas um paciente, a maioria das alterações citadas pelos autores foi observada, desta forma, lembramos a importância da procura dos profissionais mencionados para uma intervenção precoce.

\section{COMENTÁRIOS FINAIS}

Tendo em vista as dificuldades desse paciente, como exemplo, e reconhecendo que os demais também apresentam manifestações semelhantes, o atendimento fonoaudiológico realizado em nosso Ambulatório pretende abordar todas as suas possibilidades de comunicação: voz, fala, linguagem, audição, cognição, interação social levando em conta a atividade muscular facial presente e as alterações encontradas em cada um deles, dentro de todas as áreas de domínio da equipe multidisciplinar apresentada.

Todas as funções referentes ao propósito da comunicação deverão ser investigadas em profundidade, com o diagnóstico das alterações realizado e oferecimento de propostas para adaptação ou melhor condição de atuação. Para tal, são realizados exercícios oromiofuncionais, massagens intra e extraoral e estimulação de linguagem, além de incentivo ao uso de gestos. Ao término de cada terapia há uma devolutiva para os pais ou responsáveis, onde estes são orientados a dar continuidade aos exercícios em casa, reconhecer a importância da realização periódica desses e, se necessário, dirimir dificuldades.

O nosso trabalho tem se esforçado para valorizar as potencialidades individuais dessas crianças, demonstrando quer por fotos, vídeos ou mensuração, as pequenas melhoras ocorridas no dia a dia. Esta é uma tarefa extensa em que se acompanha o processo de desenvolvimento da criança como um todo, e neste caso, optamos por demonstrar o protocolo utilizado e de que forma um paciente com SM se apresenta.

\begin{abstract}
Möbius Sequence (MS) was initially described by Von Graefe, in 1888. It is characterized by palsy of VI and VII cranial nerves, determining absence of facial expression accompanied by convergent strabismus. In the children assessed in this group all described characteristics are found. However, palsy caused by other cranial nerves may also occur, impairing soft palate and tongue mobility and thus making it difficult to produce phonemes and/or showing excessive nasalization during their emission. The present study had the aim to disclose the protocol used at our institution with MS patients and to report a case from this service, as mere example. The protocol consists of two parts: anamnesis and evaluation of stomatognathic structures and functions. The anamnesis is carried out using a questionnaire responded by the caregivers, with the aim to gather information about the patient's history, since birth. The evaluation investigates mobility, motricity, muscular tonus and posture of the phonoarticulatory organs, and activities of neurovegetative functions. The muscles responsible for facial expression are also individually assessed, in order to evaluate the real possibilities for these patients' expression. To exemplify both parts of the protocol, a 10-year-old male patient was evaluated, and the results are described in this study. He has been attending therapy at this institution since 2000. With this protocol, we had the aim to identify clinical symptoms of MS related to Speech-Language Pathology, as well as to describe the individual characteristics.
\end{abstract}

Keywords: Protocols; Evaluation; Medical history taking; Möbius syndrome/diagnosis; Stomathognatic system; Case reports

\title{
REFERÊNCIAS
}

1. Momtchilova M, Pelosse B, Rocher F, Renault F, Laroche L. [Möbius syndrome: ocular and clinical manifestations]. J Fr Ophtalmol. 2007;30(2):177-82. French.
2. Canonaco RS. Sindromes genéticas com repercussões fonoaudiólogicas no recém-nascido. In: Bassetto MCA, Brock R, Wajnsztejn R. Neonatologia: um convite à atuação fonoaudiológica. São Paulo: Lovise; 1998. p. 25-57. 
3. Ferreira VJA. Síndrome de Möebius: você precisa saber! Rev Soc Bras Fonoaudiol. 1998;2(4):51-2.

4. Granato L, Pinto CF, Ribeiro MQ. Perda auditiva de origem genética. In: Lopes Filho OC, editor. Tratado de fonoaudiologia. São Paulo: Roca; c1997. p. 25-57.

5. Curtin JM. Intratemporal facial nerve lesions: infections, trauma, and new growth. Ear Nose Throat J. 1990;69(10):698-703.

6. Fagan PA. The assessment and treatment of facial paralysis. Aust Fam Physician. 1989;18(11):1400-6, 1409, 1412-6 passim.

7. Love RJ, Weeb WG. Neurology for the speech-language pathologist. 3rd ed. Boston: Butterworth-Heinemann; 1996.
8. Sánchez O, Guerra D. Síndrome de moebius: fetopatía por misoprostol: reporte de un paciente. Invest Clin. 2003;44(2):147-53.

9. Zenteno Vacheron JS, Ramos Ramirez R, Irigoyen Arroyo M, Gutierrez Cabrera JJ. Syndrome de Mobius 1. Diplejia facial congenita. Reporte de 9 casos. Rev Méd Hosp Gen Méx. 1981;44(3):102-6.

10. Pontes PAL, Behlau MS. Nasolaringoscopia. In: Altmann EBC. Fissuras labio_palatinas. São Paulo: Pró Fono; 1994. p. 25-57.

11. Guedes ZCF. Atendimento fonoaudiológico das paralisias faciais no adulto e na criança. In: Lagrota MGM. A fonoaudiologia nas instituições. São Paulo: Lovise; 1995.p.163.

Anexo 1. Protocolo de anamnese

Data: 1

Examinadora:

Nome:

Data de Nascimento: SEXO: $M(\quad) \quad F(\quad)$

End:

CEP:

Mãe:

Idade:

Pai:

Idade:

Informante:

Encaminhado por:

Hipótese Diagnóstica (HD):

Diagnosticado por:

Antecedentes Familiares:

\section{Dados da gestação:}

- Gestação:

- Como foi?

Intercorrências?

- Uso de medicamentos? ( ) Sim ( ) Não

Quando?
( ) Citotec
( ) Outros:

- Parto: ( ) Normal

( ) Cesárea

TEL:

Profissão:

- Intercorrências:

\section{Dados do nascimento:}

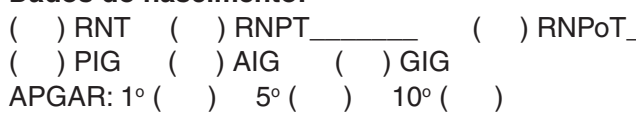

Peso ao Nascimento:

Estatura:

Idade Gestacional:

Período de Internação:

Intercorrências:

Dados do desenvolvimento:

- DNPM: Controle cervical

Sentar com apoio

Sentar sem apoio

Engatinhar

Andar

- Desenvolvimento de fala - linguagem:

Balbuciou

- Audição:

Falou as primeiras palavras

- Ouve bem? ( ) Sim ( ) Não

- Assusta com barulho? ( ) Sim ( ) Não

- Acompanha os sons ? ( ) Sim ( ) Não

- Dor de ouvido? ( ) Sim ( ) Não Otites? ( ) Sim ( ) Não

- Já realizou algum exame auditivo? ( ) Sim （）Não

Qual? 
- Histórico Alimentar:

Aleitamento Materno: ( ) Sim （）Não Tempo:

Mamadeira: ( ) Sim ( ) Não Início:

Bico: ( ) Comum ( ) Ortodôntico: $\mathrm{n}^{\circ} 1\left(\right.$ ) $\mathrm{n}^{\circ} 2($ )

Furo: ( ) Adequado ( ) Inadequado

Tipo de alimento ofertado na mamadeira:

Quem oferta alimentação:

Postura durante a alimentação:

Época das transições alimentares:
Pastoso
Semi-sólido
Sólido

Como ocorreram as transições:

Alimentação atual:

( ) VO ( ) SOG ( ) SNG ( ) Gastrostomia

-Consistência:

-Horário:

-Quantidade:

-Intercorrências:

- Engasgos freqüentes? ( ) Sim ( ) Não

Utensílios empregados para alimentação:

Quais Consistências?

Hábitos orais:

OBS:

\section{- Doenças:}

Crises convulsivas:

Doenças respiratórias:

BCP freqüente ( ) sim ( ) não Freqüência:

RGE:

Alterações cardíacas:

Má-formação craniofacial:

Alterações metabólicas:

Outras:

- Medicamentos:

- Sono:

- Historico escolar: (como está/ tipo de escola/ classe/ sociabilidade/ amigos/ preferências/ hábitos de pintar, ler)

- Internações e cirurgias

Motivo e tempo:

- Acompanhamento com outras especialidades:

- Observações da mãe:

- Observações do profissional:

- Orientações:

- Encaminhamentos: 
Anexo 2. Avaliação estrutural e funcional fonoaudiológica de órgãos fonoarticulatórios em crianças portadoras de paralisia facial congênita
Nome:
Data: / /
Idade: anos
Nasc::

Queixa:

I. Movimentação de cabeça
( ) rotação para direita
( ) rotação para esquerda
( ) para trás
( ) para frente

II. Face
( ) normal
( ) alterada
( ) simétrica
( ) assimétrica
( ) braquifacial
( ) dólicofacial
( ) mesofacial

III. Movimentação de olhos
( ) direita
( ) esquerda ( ) para cima
( ) para baixo
( ) circular para direita
( ) circular para esquerda
( ) canto superior direito
( ) canto inferior direito
( ) canto superior esquerdo
( ) canto inferior esquerdo

IV. Lábios

- posição:

( ) fechados

( ) abertos

( ) evertido

( ) encurtado

- tonicidade: ( ) normal

- freio labial: ( ) normal ( ) hipotonia

( ) curto

- mobilidade: ( ) normal

( ) alterada

- praxias orofaciais:
( ) soprar
( ) protruir
( ) retrair
( ) estalo
( ) vibração
( ) protruídos leva à direita
( ) protruídos leva à esquerda
( ) articular vogais

V. ATM

( ) normal

( ) crepitação

( ) anormal

VI. Mandíbula
( ) normal
( ) prognatia
( ) retrognatia
( ) macrognatia
( ) micrognatia
( ) simétrica

( ) assimétrica lado

- masséter e temporal: ( ) normal

- pterigoídeo medial: ( ) normal
( ) hipotonia
( ) hipertonia
( ) hipotonia
( ) hipertonia

- praxias orofaciais:

( ) abre e fecha a boca ( ) abre a boca contra resistência

( ) fecha a boca contra resistência

( ) leva para a direita ( ) leva para a esquerda ( ) leva para frente

( ) leva para trás ( ) movimentos circulares

- mobilidade: ( ) normal ( ) alterada

VII. Bochechas

( ) normal ( ) hipotonia

- mobilidade: ( ) normal

- praxias orofaciais:
( ) infla bochechas juntas
( ) infla bochecha direita
( ) infla bochecha esquerda
( ) sopra

VIII. Mentoniano
( ) normal
( ) hipotonia
( ) hipertonia

IX. Arcada dentária

- estado geral dos dentes: ( ) BEC ( ) MEC

- dentição: ( ) decídua ( ) mista ( ) permanente
( ) extranumerários
( ) $n^{\circ}$ total de dentes:

( ) apinhamentos ( ) diastema ( ) inclinação

- Arcada: ( ) normal ( ) alterada ( ) em correção ( ) classe I ～( ) classe II ( ) classe III ( ) oclusão cruzada anterior ( ) oclusão cruzada lateral ( ) aberta anterior ( ) aberta lateral ( ) topo ( ) profunda

- Linha média: ( ) sem desvio ( ) com desvio ( ) para direita

( ) para esquerda

X. Língua

- tonicidade: ( ) normal ( ) hipotonia

( ) hipertonia

- aspecto: ( ) normal ( ) geográfica

( ) fasciculada ( ) com desvio freio lingual: ( ) normal

( ) curto

- mobilidade: ( ) normal ( ) alterada 
- praxias orofaciais: ( ) para dentro e para fora ( ) estalar ( ) afinar
( ) vibrar
( ) afinar e alargar
( ) para um lado e para o outro dentro da boca
( ) para cima e para baixo dentro da boca
( ) para um lado e para o outro fora da boca
( ) para cima e para baixo fora da boca

XI. Palato
( ) normal ( ) ogival
( ) fissurado
( ) presença de fístula
( ) fissura sub mucosa
( ) atresia
( ) amplo

XII. Véu do palato
( ) normal ( ) curto
( ) ausente
( ) paralisado
( ) fissurado
( ) fissura sub mucosa
( ) movimentação normal
( ) movimentação diminuída
- úvula: ( ) normal
( ) ausente
( ) bífida
( ) sulcada

- amígdalas e adenóides:

( ) normais ( ) hipertróficas

( ) removidas

( )atróficas

XIII. Funções neurovegetativas

- Sucção

1 - líquido

$$
2 \text { - pastoso fino }
$$

3 - pastoso grosso
( ) com vedamento labial
( ) com força

( ) com ritmo

( ) fraca

( ) sem ritmo

( ) preensão labial

( ) ineficiente

( ) sem vedamento labial

( ) sem anteriorização de língua

( ) participação de bucinador

( ) com escape

( ) sem canolamento

( ) com anteriorização de língua

( ) sem participação de bucinador

- Mastigação

1 - pão francês

$$
2 \text { - waffer } 3 \text { - pão de queijo }
$$

( ) mordida anterior

( ) movimentos rotatórios

( ) mastigação bilateral

( ) mordida lateral

( ) movimentos verticalizados

( ) sem contração de masséter

( ) formação de bolo coeso

( ) empurrar o alimento com o dedo

- velocidade de mastigação: ( ) normal

( ) aumentada

( ) mastigação unilateral

( ) contração de masséter

( ) vedamento labial

( ) centralização do bolo na língua

( ) lateralização de língua

( ) bolo espalhado na $\mathrm{CO}$

( ) sem vedamento labial

( ) não - centralização

- Deglutição

1 - líquido

2 - pastoso 3 - sólido

( ) eficiente ( ) sem protrusão de língua ( ) sem movimentação de cabeça

( ) sem interposição de lábio ( ) com vedamento labial

( ) ineficiente ( ) com protrusão de língua ( ) com movimentação de cabeça

( ) com interposição de lábio

( ) sem vedamento labial

( ) sem contração de mentoniano

( ) contração de masséter

( ) sem participação de $\mathrm{mm}$ perioral

( ) propulsão do bolo eficiente

( ) com contração de mentoniano

( ) sem contração de masseter

( ) com participação de $\mathrm{mm}$ perioral

( ) propulsão do bolo ineficiente

- Respiração

$\begin{array}{lll}\text { tipo: ( ) torácica } & \text { ( ) abdominal } & \text { ( ) mista } \\ \text { modo: ( ) nasal } & \text { ( ) oral } & \text { ( ) mista } \\ & \text { ( ) ruidosa } & \text { ( ) silenciosa }\end{array}$

- Frases

O cachorro saiu na chuva;

Kiki gosta de chá;

Mamãe come mamão;

Susi piscou por causa do cisco. 